\title{
CLAVIJO OLARTE, AMPARO; RAMÍREZ GALINDO, LUZ MARIBEL. LAS PEDAGOGIAS DE LA COMUNIDAD A TRAVÉS DE INVESTIGACIONES LOCALES EN EL CONTEXTO URBANO DE BOGOTÁ. BOGOTÁ, D.C.: EDITORIAL UNIVERSIDAD DISTRITAL FRANCISCO JOSÉ DE CALDAS, 2019. 260 P.
}

\section{Daniel Calderón-Aponte*}

La reciente obra publicada por las académicas colombianas, Amparo Clavijo Olarte y Luz Maribel Ramírez Galindo, titulada Las pedagogías de la comunidad a través de investigaciones locales en el contexto urbano de Bogotá, propone la utilización de las comunidades, los entornos escolares, y las realidades socioculturales de los estudiantes como los pilares para la enseñanza y el aprendizaje de las diversas áreas del conocimiento. El trabajo exhaustivo y pertinente desarrollado por las autoras, docentes e investigadoras de la Universidad Distrital Francisco José de Caldas de Bogotá, D.C., considera entonces que, desde un ejercicio pedagógico crítico, la enseñanza debe responder a una práctica situada (KELL, 2009) en donde se articulen las comunidades y sus recursos, los entornos, y las realidades de los estudiantes con el fin de fomentar prácticas educativas más pertinentes.

El libro, de 260 páginas, toma forma de compilación dividida en tres partes que se desarrollan a través de 9 capítulos. Dentro de estos, docentes de diversas áreas del conocimiento, pertenecientes a diferentes instituciones educativas públicas de Bogotá nos comparten sus experiencias pedagógicas y de investigación, guiados por la experiencia, y en algunos casos en coautoría, de Amparo Clavijo Olarte y Luz Maribel Ramírez Galindo. Así mismo, el libro cuenta con la presentación a cargo de Iliana Reyes, investigadora mexicana del Centro de Investigación y de Estudios Avanzados del Instituto Politécnico Nacional, y un epílogo elaborado por la docente e investigadora Judy Sharkey de la Universidad de New Hampshire.

Este libro comparte los resultados de dos estudios de investigación concernientes a formación de docentes en instituciones educativas de la ciudad

\footnotetext{
* Universidad Distrital Francisco José de Caldas, Bogotá, D.C., Colombia. dcalderona@correo. udistrital.edu.co

Orcid: https://orcid.org/0000-0002-2898-6487
} 
de Bogotá. Ambos contaron con aportes académicos de índole internacional, y recibieron apoyo y financiación del Centro de Investigaciones y Desarrollo Científico de la Universidad Distrital Francisco José de Caldas. Amparo Clavijo Olarte y Luz Maribel Ramírez Galindo, por medio de su obra, nos comparten sus aprendizajes, reflexiones, e investigaciones llevadas a cabo por y con docentes de diversas instituciones educativas públicas del contexto urbano de la ciudad de Bogotá. Todas estas se fundamentan en epistemologías de la educación y formación docente que conectan el conocimiento académico de la escuela con el conocimiento que se encuentra en la comunidad, para fomentar así una educación más democrática (ZEICHNER; PAYNE; BRAYKO, 2014).

Dicho de manera sucinta, a lo largo del libro los lectores encontrarán que las autoras exploran con los demás docentes participantes los entornos escolares por medio de la indagación frente a los recursos, textos urbanos, y culturas presentes en la comunidad. Una vez identificados los posibles recursos ofrecidos dentro de estas, se propone la implementación y utilización de una pedagogía que, según Moll, Amanti, Neff, y González (1992) reconoce e incorpora las realidades, familias, fondos de conocimiento, y entornos sociales de los estudiantes. Incorporar las culturas locales e identidades de los estudiantes en el currículo escolar (SHARKEY; CLAVIJO, RAMÍREZ, 2016) resulta vital para desarrollar prácticas pedagógicas y currículos más situados y responsivos con los contextos socioculturales y las comunidades de los estudiantes.

La primera parte del libro, titulada Los maestros aprendiendo sobre la comunidad, se compone de cuatro capítulos en donde cuatro docentes investigadores, estudiantes de la Maestría en Lingüística Aplicada para la Enseñanza del Inglés de la Universidad Distrital Francisco José de Caldas, comparten sus experiencias pedagógicas e investigativas frente a la utilización de los recursos de las comunidades y los entornos escolares para fomentar la enseñanza y el aprendizaje del inglés. Como parte de su proceso de aprendizaje del componente de investigación de su maestría, los cuatro docentes investigadores parten del mapeo de sus comunidades (KRETZMANN; McKNIGHT, 1993) educativas para identificar posibles recursos que puedan responder a los estándares de formación en lenguas extranjeras y a sus respectivos currículos, para fomentar así una práctica pedagógica más pertinente y centrada en las realidades socioculturales de sus estudiantes.

La utilización de la comunidad para la articulación con el currículo y los estándares de formación en lenguas extranjeras supuso un cambio de perspectiva para los cuatro docentes que protagonizan esta primera parte. Desde este plano epistemológico, es necesario que los docentes se conviertan en agentes que indaguen 
y exploren los diversos saberes, prácticas sociales y culturales, características, y recursos que la comunidad tiene para ofrecer, con el propósito de incluirlos en sus quehaceres pedagógicos y en su currículo de lenguas (MOLL; AMANTI; NEFF; GONZÁLEZ, 1992; MURRELL, 2001). Así entonces, los cuatro capítulos presentados en la primera parte dan cuenta a su vez de cuatro experiencias investigativas en donde los docentes investigadores, utilizan la Pedagogía Basada en la Comunidad - PBC (SHARKEY, CLAVIJO, 2012) para fomentar el aprendizaje de inglés de una manera más situada al incorporar y responder a las realidades y el contexto de sus comunidades educativas. Aprenderemos, como lectores, que el inglés no sólo articula los recursos de las comunidades con el currículo y los estándares de formación en lenguas extranjeras, sino que este también se convierte en el vehículo para fomentar el desarrollo de la literacidad crítica y el aprendizaje transdisciplinar.

Bajo el nombre de Leer la comunidad críticamente en la era digital: Una mirada desde las literacidades, el capítulo uno, escrito por la docente investigadora Rosa Alejandra Medina Riveros, en compañía de las autoras Amparo Clavijo Olarte y Luz Maribel Ramírez Galindo, relata su experiencia al utilizar la comunidad y el entorno de su institución educativa como eje central para la enseñanza y el aprendizaje de inglés a través de un curso virtual.

Aquí, la docente investigadora comparte su estudio de tipo cualitativo y etnográfico en línea (KOZINETS, 2010) realizado en una universidad pública de Bogotá, en donde participaron veinticuatro estudiantes de diversos programas de pregrado. Así mismo, esta experiencia de investigación se fundamentó en tres grandes apartados teóricos: la literacidad crítica, las pedagogías comunitarias, y las multiliteracidades en la era digital. En cuanto al primer elemento teórico, el estudio se basó en la utilización de los recursos locales encontrados en la comunidad educativa para fomentar la literacidad crítica en los estudiantes de inglés. Al respecto de la literacidad crítica, Freire y Macedo (1987) señalan que el uso del lenguaje no puede encontrarse fuera de la realidad. Y precisamente, la literacidad crítica permite indagar y hacer una lectura de la realidad y el contexto en el que uno se encuentra para lograr así una transformación social. El segundo elemento teórico, la PBC (SHARKEY, CLAVIJO, 2012), se encuentra altamente relacionado con la literacidad crítica en tanto que la lectura de las comunidades permite identificar e indagar sobre las prácticas y los recursos de la comunidad, para posteriormente incorporarlos al currículo escolar.

En cuanto a los hallazgos, la docente investigadora pudo identificar dos momentos claves para el aprendizaje de inglés y el desarrollo de literacidad crítica 
por parte de sus estudiantes. En primer lugar, los estudiantes y la docente pudieron reconocer los recursos humanos, culturales, históricos, lingüísticos y ecológicos que ofrecía la comunidad universitaria. Este primer momento resultó ser revelador y fundamental para la docente y los participantes, quienes pudieron utilizar los diversos elementos y recursos presentes en la comunidad para su aprendizaje en articulación con el desarrollo de la lengua inglesa. Y, durante el segundo momento clave, los resultados sugieren que los estudiantes pudieron leer de manera crítica la comunidad universitaria al identificar problemáticas y aspectos que les afectaban como estudiantes, sin dejar de lado una actitud propositiva y de cambio frente a estas. Así entonces, los estudiantes, por medio de textos multimodales elaborados en inglés, pudieron compartir sus lecturas críticas del entorno y de la comunidad universitaria, identificando problemáticas y asumiendo una posición constructiva frente a la mismas.

El capítulo dos, titulado Las literacidades de los estudiantes de inglés desde indagaciones locales en una experiencia multimodal, escrito por la docente investigadora July Andrea Rincón Ortega en compañía de la autora Amparo Clavijo Olarte, presenta las experiencias y los resultados de una investigación-acción de tipo cualitativa, realizada en una escuela pública al sur de Bogotá. Esta contó con la participación de cuarenta estudiantes de grado décimo, cuyas edades oscilaban entre los catorce y quince años. Aquí, la docente investigadora narra y comparte cómo el entorno y la comunidad de sus estudiantes, explorado por medio de preguntas-problema, y documentado a través de textos multimodales, logra vincularse de manera pertinente a sus realidades y experiencias dentro del currículo escolar (FREIRE, 2005).

El objetivo principal de la investigación se sustenta en entender cómo las indagaciones que los estudiantes realizan sobre su comunidad pueden crear oportunidades para que se exploren diversos temas sociales y culturales dentro de sus barrios, a través de la creación multimodal desde la clase de inglés. Se entiende, entonces, lo multimodal como todo texto, que incluya imágenes, vídeos, y música, para, en este caso, representar las temáticas socioculturales de la comunidad educativa y de sus barrios. De igual modo, la docente investigadora encuentra como situación problemática la desconexión entre el currículo de lenguas y las características socioculturales de los aprendientes. Frente a esto, se adopta una perspectiva que utiliza la PBC para integrar los contenidos de la enseñanza de lengua extranjera con las indagaciones que los estudiantes puedan realizar dentro de sus comunidades (CLAVIJO, 2015b).

En cuanto a la discusión teórica, la docente investigadora logra articular de manera pertinente estudios locales y principios concernientes la utilización 
de la PBC (CLAVIJO, 2015a; MEDINA; RAMÍREZ; CLAVIJO, 2015; REYES, 2012; SHARKEY, 2012), el papel del docente comunitario (MURRELL, 2001) y la formación de maestros de lenguas desde una perspectiva socio crítica (JOHNSTON; DAVIS, 2008), y los fondos de conocimiento (MOLL, 1994) que los estudiantes traen consigo al aula de clase. Todos estos elementos teóricos y discusiones investigativas se concatenan para ofrecer un entendimiento sobre el papel fundamental que cumplen las comunidades, sus recursos, y los bagajes socioculturales de estudiantes y maestros para fomentar prácticas pertinentes que responden y sitúan el aprendizaje de lenguas extranjeras con las realidades y los contextos de los estudiantes.

Los resultados de la investigación, analizados desde del enfoque de la teoría fundamentada, permitieron revelar dos grandes temas que revelan el proceso de aprendizaje de los estudiantes. Primero, los estudiantes pudieron indagar sobre los diversos temas de su interés dentro de la comunidad $\mathrm{y}$, por medio de reportes multimodales que incluían fotos, vídeos, entrevistas, canciones, entre otros, pudieron identificar los recursos presentes en la comunidad y documentar situaciones problemáticas dentro de la misma. Segundo, la utilización de redes sociales, como Facebook, resultó ser una herramienta fundamental para el aprendizaje y la interacción de los estudiantes en inglés.

Así entonces, por una parte, los resultados sugieren que, a partir de indagaciones dentro de la comunidad, los estudiantes se convierten en sujetos activos e investigadores que formulan preguntas a situaciones problemáticas y de interés dentro de sus barrios. La creación de textos multimodal, elaborados y presentados en inglés, resulta ser una herramienta importante para la divulgación y socialización de las indagaciones de los estudiantes dentro de la comunidad. Y, por otra parte, las redes sociales y los blogs en línea se convierten en elementos pertinentes para fomentar el aprendizaje y el desarrollo de las competencias lingüísticas al crear colaborativamente textos y discusiones que utilizaban el inglés para dar sentido a sus comunidades.

La indagación sobre el refrigerio escolar para construir convivencia en la clase de inglés, escrito por la docente investigadora Anna Janeth Gómez Gutiérrez, se enmarca en el capítulo tres de este libro. La docente, parte de la noción de que es posible aprender inglés mientras se aprende del entorno y del mundo en sí, utilizando esta lengua como un medio para fomentar dicho proceso de aprendizaje.

En esta investigación, desarrollada en un colegio público ubicado al suroccidente de la ciudad, participaron los estudiantes del grado quinto de primaria. Partiendo de utilización de la comunidad como vehículo para establecer conexiones 
con el currículo escolar (MEDINA; RAMÍREZ; CLAVIJO, 2015), la docente investigadora implementó y desarrolló fases de indagación (SHORT; HARSTE; BURKE, 1996) que respondían a los estándares de enseñanza de inglés y de ciencias naturales del Ministerio de Educación Nacional de Colombia, con el fin de indagar sobre el refrigerio escolar dentro de esta institución educativa. Adicionalmente, se logró fomentar un aprendizaje transdisciplinar desde las áreas de ciencias e inglés, al vincular procesos de indagación frente al refrigerio que allí se les brindaba.

Siguiendo lo propuesto por Short, Harste, y Burke (1996), la investigación siguió una serie de etapas para la indagación. En primer lugar, los estudiantes conformaron sus grupos de investigación según sus propios criterios. En segundo lugar, exploraron su comunidad escolar con el fin de investigar acerca de todo lo relacionado con el refrigerio escolar, incluyendo vocabulario clave en inglés, y las posibles fuentes de consulta para el desarrollo de sus indagaciones. Después, en tercera instancia, aprendieron a hacer preguntas en inglés y español que guiaron sus respectivas investigaciones en torno a los refrigerios. Posteriormente, una vez construidas las preguntas base para el desarrollo de su ejercicio investigativo, la cuarta etapa consistió en la recolección de la información. Esta se pudo recopilar desde diversas fuentes, las cuales incluían consultas en internet, libros y revistas especializadas, y entrevistas a distintas personas pertenecientes a la comunidad escolar.

Una vez recolectada la información que daría respuesta a sus preguntas de investigación, la quinta fase se enfocó en la organización de la información colectada, y la evaluación de su pertinencia para el desarrollo del ejercicio de indagación. Finalmente, la sexta fase consistió en compartir los resultados preliminares de la investigación en torno al refrigerio escolar. Por medio de la ayuda de la docente investigadora, los estudiantes crearon libros con textos e imágenes en inglés para divulgar sus aprendizajes. Los resultados finales se compartieron en un evento dentro de la biblioteca del colegio. Allí, los estudiantes de quinto grado pudieron dar a conocer su experiencia a toda la comunidad educativa como investigadores que lograron articular las áreas de ciencias naturales y el inglés, con el fin de explorar e indagar sobre todo lo concerniente al refrigerio ofrecido dentro del colegio.

La docente investigadora recalca que, más allá de fomentar un aprendizaje transdisciplinar que respondiera a los estándares propuestos por las autoridades educativas del país, esta experiencia de investigación e implementación pedagógica basada en la indagación permitió una reflexión constante entre los estudiantes de quinto grado, lo cual no sólo mejoró su aprendizaje colaborativo, sino también la convivencia y la resolución de problemas dentro del aula. Así mismo, durante 
el proceso de indagación sobre el refrigerio escolar, los estudiantes no sólo desarrollaron actitudes de investigación que se articularon con el aprendizaje de ciencias naturales, también se pudo evidenciar el desarrollo de aprendizaje de lengua inglesa acudiendo al translanguaging (GARCÍA, 2009) al utilizar de manera indistinta la lengua materna y el inglés para dar sentido a su uso y desarrollo. En conclusión, la indagación sobre el refrigerio escolar permitió desarrollar una perspectiva sociocultural de la educación, en la cual las comunidades resultan indispensables como recursos para concatenar el trabajo y el aprendizaje de diversas áreas del conocimiento.

En el último capítulo de la primera parte, la docente investigadora Nhora Lucía Reyes Navarro nos presenta su investigación titulada Hacia una educación comunitaria en el aula de inglés con niños en condición vulnerable. Geográficamente hablando, esta investigación se desarrolló en un barrio ubicado al noroccidente de la capital colombiana, y contó con la participación de veinticinco estudiantes de entre cinco y nueve años, pertenecientes a un programa de educación no formal. Estos, a su vez, se encontraban en condición de vulnerabilidad debido al perfil y contexto socioeconómico de sus familias. La investigación se llevó a cabo por medio de la creación de una unidad curricular que consistía en cinco lecciones, para así integrar el desarrollo de la literacidad, el aprendizaje de inglés, y las experiencias de vida de los estudiantes.

En cuanto a las consideraciones y debates teóricos, la docente investigadora utiliza la $\mathrm{PBC}$ como una perspectiva alternativa de educación que incorpora y responde a los recursos locales presentes en las comunidades, con el fin de crear y proponer currículos más pertinentes para las realidades socioculturales de los estudiantes. Empero, para que esto sea posible, los programas y la formación de docentes resultan ser vitales para promover una práctica reflexiva frente a la importancia que adquiere la incorporación y utilización de los recursos de la comunidad en donde se enseña (SCHECTER, SOLOMON; KITTMER, 2003). De igual manera, perspectivas locales (CLAVIJO, 2015a; SHARKEY; CLAVIJO; RAMÍREZ, 2016) señalan que los docentes pueden identificar y utilizar los diversos recursos disponibles dentro de las comunidades para fomentar así maneras más relevantes de enseñanza y aprendizaje de las lenguas extranjeras. Los docentes, por ende, se convierten en maestros comunitarios que, según Murrell (2001), conocen las prácticas, realidades, contextos y recursos de la comunidad, en donde ejercen su quehacer pedagógico.

Los resultados de esta implementación pedagógica fundamentada en la comunidad sugieren que, durante las cinco lecciones y actividades implementadas, 
los estudiantes pudieron comprender y leer su comunidad frente a las problemáticas sociales que allí ocurren. De igual manera, el inglés se convirtió en el vehículo para que, a través de este, los estudiantes pudieran reflexionar sobre sus proyectos de vida y sus contextos comunitarios.

Los cuatro primeros capítulos nos muestran cuatro experiencias pedagógicas e investigativas de docentes de inglés en su proceso de formación de maestría. Estas se desarrollaron en los contextos educativos donde los docentes investigadores ejercen su quehacer pedagógico. Es menester resaltar que estas experiencias se fundamentan en la utilización de la comunidad para fomentar prácticas y el desarrollo de la literacidad crítica para leer e identificar los diversos recursos y las problemáticas presentes dentro de sus comunidades. Esto supone un cambio epistemológico al considerar que la enseñanza y el aprendizaje de inglés parte de una práctica contextualizada que incorpora y responde los aspectos socioculturales, lingüísticos, y las comunidades de los estudiantes. El inglés, entonces, deja de ser el objeto de estudio per se y se convierte ahora en un vehículo que articula el desarrollo de la literacidad crítica con las realidades y los contextos de los aprendientes.

En la segunda parte del libro, titulada Los formadores que aprenden de los maestros y sus comunidades, las autoras presentan, a modo de introducción, sus experiencias y posturas teóricas como resultado de su trabajo como formadoras de docentes en tres instituciones educativas públicas de la ciudad de Bogotá. Esta experiencia de formación docente se desarrolló bajo el proyecto de investigación Local literacies as critical resources in teacher education: Local and global impacts, cuyo principal propósito consistía en inquirir y aprender cómo desde las áreas de ciencias naturales, sociales, educación física, lenguaje, y química, los docentes pueden articular la Pedagogía Basada en la Comunidad (SHARKEY; CLAVIJO, 2012) dentro de sus currículos y cotidianidades de los estudiantes.

Las autoras sostienen que los programas de formación docente deben tener como eje central la comprensión relacional entre lo local y lo global. De esta manera, los docentes pueden construir currículos más pertinentes que vinculan las realidades, las comunidades, y las vidas de sus estudiantes. Como resultado, los docentes dejan de ser sujetos pasivos para convertirse en productores activos de conocimiento (COCHRAN-SMITH, LYTLE, 1993), los cuales observan sus comunidades educativas para identificar los diversos recursos allí presentes (MEDINA, RAMÍREZ, \& CLAVIJO, 2015).

En cuanto al desarrollo metodológico de este ejercicio investigativo y de formación docente, las autoras, por medio de la participación y colaboración activa de los docentes de las tres instituciones, implementaron cuatro talleres para guiar los 
principios conceptuales y prácticos. El mapeo y conocimiento de las comunidades fue, una vez más, el inicio y eje central para el desarrollo del proyecto. Esto resultó fundamental para la creación de prácticas pedagógicas y currículos que apreciaran e incorporaran los recursos disponibles dentro de las comunidades escolares.

Así entonces, la segunda parte del libro nos presenta tres capítulos de experiencias pedagógicas e investigativas de docentes desde diversas áreas del conocimiento, quienes, al igual que sus pares presentados en el primer capítulo, pudieron incorporar los recursos existentes dentro de sus comunidades para fomentar sus prácticas de enseñanza y aprendizaje. Aprenderemos de las experiencias y las voces de estos docentes que, en coautoría con Amparo Clavijo Olarte y Luz Maribel Ramírez Galindo, han apostado por la renovación de sus prácticas pedagógicos para hacer sus clases y currículos más situados y responsivos con las realidades y los contextos de sus estudiantes.

El capítulo cinco, el primero que se encuentra en este segundo gran apartado, se titula Las realidades sociales y culturales de los barrios de la localidad de San Cristóbal: una opción para indagar a través del Simonu en el Colegio El Rodeo, y es escrito por los docentes Víctor Hugo Chacón, Martha Liliana Gutiérrez Herrera, Gloria Pulido Becerra, Luz Paola Rincón Jiménez, y July Andrea Rincón Ortega. Los docentes pertenecientes al Colegio El Rodeo, una institución de educación pública ubicada al suroriente de Bogotá, conforman un grupo de trabajo interdisciplinario desde las áreas de inglés, ciencias naturales, ciencias sociales, y educación física para abordar las diversas problemáticas presentes en los barrios de su comunidad.

El proyecto en esta institución inició con el mapeo y descubrimiento de la comunidad (KREZTMANN \& McKNIGHT, 1993) y el entorno escolar por parte de los docentes, para posteriormente proponer temáticas a trabajar desde sus áreas del conocimiento. Se trabajó con dos grupos de estudiantes conformados por cuarenta y un estudiantes de décimo grado y treinta y cuatro estudiantes de cuarto grado. Frente al primer grupo, los docentes de ciencias sociales, educación física, e inglés, proponen investigar con sus estudiantes a partir de sus experiencias dentro de la comunidad para fomentar así el desarrollo y fortalecimiento de su pensamiento crítico. Para el segundo grupo de estudiantes, los docentes proponen desarrollar un proyecto desde el área de ciencias naturales para indagar sobre los posibles problemas medioambientales y sus impactos en la comunidad.

El componente metodológico del proyecto resultó fascinante. Se optó por la simulación de un modelo de las Naciones Unidas (Simonu), el cual es una propuesta pedagógica para integrar instituciones educativas de carácter público y privado para el desarrollo del pensamiento crítico por medio de un ejercicio de deliberación. Tal 
como acontece en las asambleas y sesiones de las Naciones Unidas, en donde los países cuentan con una representación, los estudiantes representan sus respectivos barrios y, de manera crítica e informada, presentan temáticas concernientes a las problemáticas de sus contextos. Así entonces, siguiendo el modelo de deliberación propuesto en el Simonu, los docentes y estudiantes propusieron dos grandes líneas de trabajo, una correspondiente a cada grado. Para el grado décimo, se propuso abordar las problemáticas de los barrios de la comunidad y, para el grado cuarto, el manejo de residuos sólidos. Ambos proyectos y líneas de trabajo respondían, de igual manera, a los lineamientos de aprendizaje de cada una de las áreas del conocimiento participantes.

El trabajo de indagación con los estudiantes de cuarto y décimo grado se desarrolló en tres momentos principales: primero, el reconocimiento de su contexto y las problemáticas de su comunidad, segundo, la construcción de textos y, tercero, la preparación para el Simonu. Durante el primer momento, los estudiantes de décimo grado iniciaron con el mapeo y el registro fotográfico de los barrios de su comunidad. Este fue compartido y socializado mediante la creación de presentaciones en inglés y español. A su vez, los estudiantes de cuarto grado también realizaron un recorrido y mapeo de su comunidad en compañía de los padres de familia. Durante este, los estudiantes pudieron tomar fotografías y hacer preguntas a distintas personas de la comunidad con el fin de identificar los problemas ambientales allí presentes. Como resultado, pudieron descubrir que la mala disposición y utilización de residuos era, entre otros, el principal problema que afectaba a la comunidad.

Para el segundo momento, desde el área de ciencias sociales, los estudiantes de décimo grado crearon un portafolio que contenía la información concerniente a los aspectos geográficos, socioeconómicos, históricos, políticos, ambientales, y culturales de los respectivos barrios de la comunidad. Este se desarrolló a partir del proceso de indagación e investigación de los aspectos anteriormente mencionados de cada barrio. En cuanto a los estudiantes de cuarto grado, igualmente, mediante la creación de portafolios, el proceso de indagación se basó en el uso, procedencia e impacto, y posible reciclaje de los residuos identificados dentro de la comunidad. Con toda la información recolectada a través de sus indagaciones y el trabajo posterior, ambos grupos elaboraron textos y discursos de apertura para presentar sus hallazgos concernientes a las problemáticas presentes en los distintos barrios de la comunidad. Previo a la socialización de sus resultados e inquietudes durante el Simonu, los estudiantes realizaron plenarias preparatorias para familiarizarse con las dinámicas, protocolos, el lenguaje utilizado, las intervenciones y debates presentes en las asambleas de las Naciones Unidas. 
Finalmente, durante el tercer momento, los estudiantes de ambos grados llevaron a cabo su respectiva simulación de una asamblea de las Naciones Unidas (Simonu). Esta, a su vez, se dividió en tres momentos en los cuales los estudiantes, primero, hicieron la presentación de los participantes y la naturaleza del debate, segundo, la presentación de cada presidente de mesa y, tercero, presentaron los discursos de apertura que, para el caso de décimo grado, fueron en inglés y en español. Los debates del Simonu en cuarto grado se generaron alrededor de los residuos de la comunidad. Esto permitió un ejercicio reflexivo para discutir acerca de temas ambientes presentes en su comunidad con el fin de fomentar hábitos de cuidado del medio ambiente. Entretanto, el debate en décimo grado se basó en tres grandes problemáticas identificadas dentro de la comunidad: inseguridad, contaminación a causa de las basuras, y el consumo de psicoactivos.

A modo de conclusión, los docentes de la institución educativa El Rodeo, pudieron concebir la utilización de la $\mathrm{PBC}$ como un enfoque que permitió conectar el currículo escolar con las comunidades de los estudiantes. Por medio del modelo y las dinámicas del Simonu, los estudiantes de ambos grados se convirtieron en investigadores y sujetos activos que no sólo aprenden de sus comunidades, sino que también identifican problemáticas allí presentes y proponen de una manera reflexiva e informada soluciones para el desarrollo de sus distintos barrios. El Simonu y la incorporación de las realidades y el contexto de los estudiantes se convirtieron en medios pertinentes para que los estudiantes pudieran desarrollar aprendizajes, competencias argumentativas, críticas, y reflexivas desde las indagaciones locales dentro de los barrios de su comunidad.

El capítulo seis, bajo el nombre de Estudio de las fuentes de contaminación en el entorno escolar que afectan el medio ambiente en el Colegio Codema: acciones de los estudiantes para la comunidad, nos presenta la experiencia pedagógica innovadora y de investigación de tres docentes en el Colegio Codema, una institución de educación pública ubicada en el suroccidente de la capital colombiana. Aquí, los docentes Ana Janeth Gómez Gutiérrez, Carlos Felipe Torres Basante, y Ana Isabel Cabra, trabajan con los estudiantes de quinto, décimo, y undécimo grado y con ellos se proponen indagar sobre los recursos que ofrece la comunidad y las problemáticas también allí presentes. Se identificaron, como resultado, dos grandes temáticas relevantes para el desarrollo de esta experiencia pedagógica: la contaminación y el uso de agua en la comunidad.

Desde una pedagogía que es responsiva con la comunidad, los docentes explican que tanto maestros y estudiantes crean de manera conjunta el currículo. De este modo, los estudiantes se convierten en actores fundamentales que, al 
conocer los entornos y contextos comunitarios, aportan temáticas y problemáticas pertinentes que son incluidas en el desarrollo y trabajo curricular. Así entonces, la implementación de las pedagogías que celebran e incluyen las comunidades dentro de sus ejes temáticos, permite la articulación entre las diversas áreas del conocimiento, y la expansión de los procesos de aprendizaje fuera del aula de clase. Aprenderemos, por medio de la experiencia de estos docentes que, la utilización de la comunidad para el quehacer pedagógico contribuye a la concepción y creación de los currículos desde perspectivas que liberan y humanizan (FREIRE, 2005).

Partiendo de una pregunta generadora encaminada a determinar el nivel de contaminación presente en el entorno de la comunidad, los docentes desde el área de lenguaje, inglés y humanidades crearon unidades curriculares fundamentadas en las diversas de la investigación-acción propuestas por Kemmis y McTaggart (1988). Así entonces, durante la primera fase de exploración, los estudiantes, los docentes y algunos padres de familia, realizaron el mapeo y reconocimiento de la comunidad para documentar e identificar los recursos disponibles dentro de la comunidad (KRETZMANN \& McKNIGHT, 1993). Posteriormente, en la segunda fase, donde se prioriza la información y las problemáticas identificadas, los estudiantes y docentes determinaron que la contaminación en la comunidad era un aspecto relevante para su futura indagación. Durante esta fase, se pudo consolidar la temática a desarrollar y la metodología para abordar esta dentro de la comunidad.

La tercera fase, concerniente a la indagación, abarcó la recolección de información por parte de los estudiantes. Estos, en una actitud investigativa, pudieron ahondar por medio de encuestas y entrevistas dentro de la comunidad, las problemáticas previamente identificadas en la primera fase del proyecto. Durante la siguiente fase, los estudiantes socializaron los procesos, resultados y productos de sus indagaciones locales dentro y fuera del aula de clase. Por una parte, pudieron socializar con sus demás compañeros de curso sus resultados y, por otra parte, compartieron folletos con los demás cursos y miembros del colegio durante una feria científica. Finalmente, durante la quinta y última fase de evaluación de resultados, los tres docentes participantes pudieron compartir y socializar su experiencia pedagógica y de investigación en el último taller del proyecto de formación de docentes liderado por las autoras Amparo Clavijo Olarte y Luz Maribel Ramírez Galindo.

En el capítulo siete del libro, titulado Una mirada al entorno y las manifestaciones estético-políticas de los jóvenes en la comunidad Alfonsina, la docente investigadora y autora Luz Maribel Ramírez Galindo junto con la docente Aura Inés Cerrón Correa, relatan y comparten dos experiencias de investigación e implementación pedagógica que 
desarrollaron en el Colegio Alfonso López Pumarejo, su institución educativa ubicada al suroccidente de Bogotá.

Las docentes, de entrada, contextualizan la extensa trayectoria y relación que su colegio ha tenido con la Universidad Distrital, en la cual se han podido desarrollar proyectos de investigación de manera transdisciplinar que incorporan pedagogías y currículos centrados en la comunidad y los contextos de los estudiantes. Este vínculo entre ambas instituciones ha generado un impacto positivo dentro de los docentes de este colegio, ya que buscan de manera constante nuevos mecanismos y estrategias para fomentar un aprendizaje que responda al contexto socio histórico de los estudiantes y de la comunidad y los entornos del colegio.

Fruto de esta relación institucional, el grupo de docentes del Colegio Alfonso López Pumarejo ha podido desarrollar proyectos de investigación innovadores y relevantes para el contexto de los estudiantes. Los docentes, entonces, se convierten en actores activos en una búsqueda constante por brindar alternativas dentro del proceso educativo dentro de la institución. Al respecto, las docentes Luz Maribel Ramírez Galindo y Aura Inés Cerrón Correa relatan y ejemplifican los diversos proyectos anteriores que, basados en la $\mathrm{PBC}$ y una constante indagación, utilizaron el entorno educativo y la comunidad para articular saberes desde las diversas áreas del conocimiento con los contextos y las realidades de los estudiantes. Empero, más allá de la descripción pertinente de los proyectos e investigaciones desarrollados anteriormente en esta institución producto del vínculo con la Universidad Distrital, las autoras de este capítulo nos presentan dos nuevos proyectos que alimentan ese espíritu constante de investigación de la comunidad Alfonsina.

En el primer proyecto, Visibilización de las subjetividades y la participación estéticapolítica de los jóvenes de grado once, tiene como objetivo comprender de qué manera las diversas experiencias artísticas, culturales, democráticas, y deportivas, presentes dentro y fuera del colegio, generan prácticas y visiones de apropiación del mundo de los estudiantes como sujetos políticos. Este proyecto, en donde participan los estudiantes de grado once, busca entonces una comprensión de la dimensión estética de estos jóvenes para determinar el rol que esta desempeña en concordancia con la política para la configuración de sus subjetividades.

Para tal fin, y por medio de talleres alrededor de la política, liderazgo, derechos humanos, normas institucionales, uso de los uniformes, y el gobierno escolar, el proyecto se propuso como objetivo de aprendizaje fomentar en los estudiantes su propio reconocimiento como sujetos políticos por medio de su participación política y estética para configurar así sus subjetividades. Esta sensibilización y autorreconocimiento por parte de los estudiantes de grado once les permitió 
exponer y representar las diversas culturas juveniles existentes en los barrios, y sus intereses y preferencias por los estilos musicales. Así mismo, los estudiantes pudieron identificar que ciertos aspectos de su vestimenta actual configuran su adhesión a determinados grupos culturales a los que admiran o pertenecen.

El segundo proyecto presentado por las autoras de este capítulo de una manera más sucinta lleva por nombre Mi entorno, un espacio de aprendizaje sobre tecnología de alimentos. Desarrollado en seis semanas con estudiantes de décimo grado desde el área de tecnología de alimentos, se realizaron recorridos por la comunidad y el entorno cercano al colegio para documentar e identificar por medio de fotografías, entrevistas, y encuestas, las industrias alimenticias allí presentes. Esto con el fin de determinar los distintos procesos industriales químicos relacionados con el procesamiento de alimentos. Este proyecto se propuso como objetivo general la identificación y el análisis de las diversas industrias alimenticias presentes en la comunidad escolar para conocer acerca de sus evoluciones tecnológicas y sus posibles aportes como contextos de aprendizaje.

La implementación de este proyecto permitió a las docentes evidenciar el potenciamiento del currículo escolar al valorar e incorporar los recursos identificados dentro de la comunidad y el entorno del colegio. Esto fomentó, a su vez, el desarrollo de prácticas metodológicas, didácticas, y pedagógicas más contextualizadas. Y, de igual manera, fue posible generar intercambios de saberes en distintos planos entre las familias, los estudiantes, los docentes, y otros actores dentro de la comunidad.

A modo de conclusión de la segunda parte, las autoras e investigadoras, Amparo Clavijo Olarte y Luz Maribel Ramírez Galindo, toman la palabra nuevamente. Relatan entonces que, dentro de su experiencia como formadoras de docentes de inglés, han podido aprender con desde y con los docentes la exploración y la utilización de los recursos existentes en las comunidades para fomentar la enseñanza y el aprendizaje en todas las áreas curriculares. Los tres capítulos presentados en esta segunda parte, brindan experiencias pedagógicas e investigativas exitosas que lograron articular diversos campos del conocimiento desde las ciencias naturales, sociales, educación física y lenguaje, con las comunidades, los estudiantes, las familias, y los entornos de las instituciones educativas.

El vínculo entre las distintas instituciones educativas y la Universidad Distrital, permitió por medio de un trabajo colaborativo aprender mutuamente acerca de las maneras para vincular la enseñanza y el aprendizaje escolar con los recursos y fondos de conocimiento locales presentes en los contextos y cotidianidades de los estudiantes. El reconocimiento y la utilización de los recursos presentes en la comunidad resultan cruciales para que los docentes, como punto de 
partida, articulen y transformen sus prácticas pedagógicas con el fin de establecer conexiones entre los contenidos curriculares y sus estándares, con las realidades del entorno de los estudiantes.

La tercera y última parte del libro, que se titula Reflexiones sobre pedagogías situadas y literacidades críticas que resignifican la formación docente, presenta el capítulo ocho donde se discuten los resultados, y el capítulo nueve en donde se propone una discusión crítica para la formación de maestros de lenguas frente a la situación de las lenguas minoritarias en la ciudad de Bogotá. En esta última sección, las autoras reflexionan sobre los resultados y las implicaciones que tiene la implementación de los proyectos y las investigaciones que los docentes pudieron desarrollar por medio de la PBC para resignificar la enseñanza de las diversas áreas del conocimiento, al utilizar las literacidades críticas para leer y descubrir los recursos presentes en las comunidades y entornos escolares.

En el capítulo ocho, Discusión de los resultados, se presentan las consideraciones, aprendizajes, implicaciones, y reflexiones de las autoras a partir de las dos investigaciones aquí presentadas en el libro. El trabajo de formación e investigación con los diversos docentes en el contexto urbano de Bogotá permitió la utilización pedagógica de los recursos de las comunidades para incorporarlos en el aula y en el currículo escolar. Las literacidades locales fueron tomadas, también, como recursos pertinentes para la articulación pedagógica, y el desarrollo de la literacidad crítica permitió que estudiantes, docentes, y padres de familia, pudiesen leer sus comunidades para indagar, identificar, y proponer soluciones a problemáticas allí presentes. Tras el análisis de los resultados, las autoras pudieron establecer dos grandes categorías: la primera denominada Del discurso deficitario a la acción pedagógica transformadora, y la segunda bajo el nombre de Pedagogías situadas y literacidades críticas que resignifican la enseñanza del lenguaje.

En cuanto a la primera categoría emergente, los resultados sugieren que, por medio de sus proyectos, los docentes pudieron superar las visiones remediales y deficitarias de la educación que concibe a los estudiantes como sujetos carentes de conocimiento. Más allá de esta noción perversa de la educación tradicional, los resultados de este libro sugieren que las implementaciones pedagógicas, las investigaciones, y las experiencias de los docentes presentados a lo largo de los capítulos, dan cuenta de su transformación epistemológica y práctica. Se cuestiona entonces la noción deficitaria y bancaria de la educación y se pasa a promover la indagación, valoración, e incorporación de los recursos locales de las comunidades para fomentar desde estos el currículo de las diversas áreas del conocimiento. La educación, entonces, se convierte en una práctica situada que resignifica y responde 
a los saberes y recursos de las comunidades de los estudiantes. Sin embargo, para que esto sea posible, las autoras resaltan la importancia que tiene la promoción de un enfoque que utilice la $\mathrm{PBC}$ dentro de los programas de formación docente fomentar vínculos entre la enseñanza, el currículo, el aprendizaje, con las realidades y los recursos locales de las comunidades.

La segunda categoría se relaciona con el desarrollo de las literacidades críticas para que los docentes pudiesen leer sus comunidades para identificar recursos, problemáticas, y posibles soluciones. Después de este ejercicio crítico de lectura y descubrimiento de sus comunidades, los docentes pueden proponer prácticas pedagógicas y crear currículos que incorporen los entornos y sus recursos como aspectos centrales para fomentar el aprendizaje desde diversas áreas disciplinares. Así entonces, tal como lo proponen Freire y Macedo (1987), el uso del lenguaje está relacionado con la realidad. Por lo tanto, las autoras también sugieren que las prácticas pedagógicas que se sitúan en las comunidades y realidades de los estudiantes pueden fomentar el desarrollo de la literacidad crítica mediante una lectura activa y consciente de sus contextos, para así identificar problemáticas en sus realidades y entornos con el fin de proponer posibles soluciones.

La docente investigadora, Amparo Clavijo Olarte, concluye la tercera parte con el capítulo titulado Repensar la formación docente desde el caso de los niños Embera en Bogotá. Aquí, la autora comparte su reflexión a partir de una experiencia de investigación con un grupo de niños indígenas Embera en una escuela pública del centro de Bogotá. Desde una postura crítica, la autora se indaga por el estado de la educación de niños y familias indígenas que llegan en condición de desplazamiento a las aulas públicas de la ciudad, y asevera que la educación de los grupos minoritarios, como los pueblos indígenas, nos concierne a todos los docentes y formadores en Latinoamérica.

Utilizando ciertos elementos teóricos e investigaciones que documentan estrategias para fomentar la educación de las poblaciones indígenas en las escuelas (MEJÍA, 2006), la lengua como un derecho de las poblaciones minoritarias (RUIZ, 1984), y la situación de los pueblos indígenas en Suramérica (HORNBERGER, 2000), entre otras, la autora propone indagar sobre la manera en que los formadores y los docentes pueden educar teniendo en cuenta y respondiendo a la diversidad sociocultural, étnica, y lingüística de las poblaciones minoritarias.

En cuanto al desarrollo del estudio, la autora señala que este se basó en la PBC con los niños Embera para integrar sus contextos y conocimientos socioculturales al currículo propuesto desde la escuela, con el fin de ampliar una visión de educación monolingüe. En otras palabras, se pudo determinar que la enseñanza del inglés 
para los niños Embera no resultaba relevante en tanto que este no tenía función alguna en sus nuevas vidas en la ciudad de Bogotá. Para esto, se contó con una implementación pedagógica con el fin de apreciar y posicionar la lengua indígena Embera dentro de la escuela como un recurso fundamental para el aprendizaje del español.

Esto significó un cambio frente a la política educativa concebida dentro de la institución puesto que se reconoció como una escuela multicultural y multilingüe debido a las características de todos sus estudiantes. Así mismo, por medio de este estudio y su respectiva implementación pedagógica, Amparo Clavijo Olarte resalta la importancia de implementar políticas y acciones para la revitalización de las lenguas nativas e indígenas del país, para cuestionar así las políticas educativas tradicionales y dominantes que poco o nada hacen para promover el estudio, uso, y preservación de las lenguas minoritarias en situación de riesgo.

En conclusión, las docentes investigadoras Amparo Clavijo Olarte y Luz Maribel Ramírez Galindo, junto con todos los docentes de las diversas instituciones educativas, presentan de una manera pertinente sus investigaciones, implementaciones pedagógicas, experiencias, reflexiones, y aprendizajes al utilizar las Pedagogías Basadas en la Comunidad. Por medio de estas, fue posible identificar e incorporar literacidades y recursos presentes en los entornos escolares para fomentar una educación situada y responsiva con las realidades y los contextos socioculturales de todos los estudiantes. Más allá de responder a estándares y currículos que, en su gran mayoría, parecen omitir los contextos y las realidades de los estudiantes y sus comunidades, vemos que las experiencias de los docentes aquí presentes muestran casos exitosos en donde las diversas áreas del conocimiento aprecian los diversos saberes y recursos presentes dentro de las comunidades para desarrollar desde allí su quehacer pedagógico.

Escribo ahora desde mi locus de enunciación como docente de inglés para invitar a mis demás colegas lectores para descubrir, valorar, apreciar, e identificar los diversos recursos presentes en las comunidades para fomentar prácticas de enseñanza y aprendizaje más pertinentes y situadas. Aprenderán los lectores de Las pedagogías de la comunidad a través de investigaciones locales en el contexto urbano de Bogotá, al igual que yo he podido aprender, que la utilización y apreciación de las comunidades se convierten en recursos pedagógicos que contribuyen de manera significativa para superar nociones y discursos tradicionales de la educación desde una perspectiva deficitaria. Educar es un acto de compromiso, cambio, amor, y de resistencia. Aprendamos de las comunidades donde nos desempeñamos como docentes para, desde allí, resistir a prácticas impuestas con el fin de fomentar aprendizajes más 
pertinentes y responsivos con los contextos socioculturales, lingüísticos, históricos, y políticos de nuestros estudiantes.

\section{REFERENCIAS}

CLAVIJO, A. (2015a). Implementing Community Based pedagogies with teachers in Colombia to enhance the English curriculum. En M. Perales y M. Méndez (Eds.), Experiencias de docencia e investigación en lenguas extranjeras (pp. 31-43). Chetumal, México: Editorial Universidad Quintana Roo.

CLAVIJO, A. (2015b). Research tendencies in the teaching of English as a foreign language. Colombian Applied Linguistic Journal, 17(1), 5-10.

COCHRAN-SMITH, M.; LYTLE, S. L. (1993). Inside/outside: Teacher research and knowledge. New York: Teachers College Press.

COMBER, B.; KAMLER, B. (2004). Getting out of deficit: Pedagogies of reconnection. Teaching Education, 15(3), 293-310.

FREIRE, P. (2005). Pedagogía del oprimido. Coyoacán, México: Siglo XXI.

FREIRE, P.; MACEDO, D. (1987). Literacy: Reading the word and the world. South Hadley: Bergun \& Garvey.

GARCÍA, O. (2009). Education, multilingualism and translanguaging in the 21st Century. En Social justice througb multilingual education (pp. 140-158). Tonawanda, NY: Multilingual Matters.

HORNBERGER, N. H. (2000). Bilingual education policy and practice in the Andes: Ideological paradox and intercultural possibility. Antbropology and Education Quarterly, 2(31), 173-201.

JOHNSTON, R.; DAVIS, J. (2008). Negotiating the dilemmas of community-based learning in teacher education. Teaching Education, 19(4), 351-360.

KELL, C. (2009). Situar las prácticas: Nuevos estudios de cultural escrita y estudios etnográficos de Sudáfrica. En J. Kalman y B. Street (Eds.), Lectura, escritura y matemáticas como prácticas sociales: Diálogos con América Latina. México: Siglo XXI.

KEMMIS, R.; MCTAGGART, V. (1988). The action research reader (3rd ed.). Geelong, Victoria: Deakin University Press. 
KOZINETS, R. V. (2010). Netnograpby: Doing etbnograpbic research online. Thousand Oaks, CA: Sage.

KRETZMANN, J. P.; MCKNIGHT, J. L. (1993). Building communities from the inside out: A patb toward finding and mobilizing a community's assets. Chicago, IL: ACTA Publications. Recuperado de http://www.abcdinstitute.org/publications/downloadable/

MEDINA, R. A.; RAMÍREZ, L. M.; CLAVIJO, A. (2015). Reading the community critically in the digital age: A multiliteracies approach. En P. Chamness Miller, M. Mantero y H. Hendo (Eds.), ISLS readings in language studies (vol. 5, pp. 45-66). Grandville, MI: International Society for Language Studies.

MEJÍA, A. M. de (2006). Bilingual education in Colombia: Towards recognition of languages, cultures and identities. Colombian Applied Linguistics Journal, 8, 152-168.

MOLL, L. (1994). Literacy research in community and classrooms: A sociocultural approach. En R. B. Ruddell, M. P. Ruddell y H. Singer (Eds.), Theoretical models and processes of reading (4th ed., pp. 179-207). Newark: International Reading Association.

MOLL, L. C.; AMANTI, C.; NEFF, D.; GONZÁLEZ, N. (1992). Funds of knowledge for teaching: A qualitative approach to connect households and classrooms. Theory into Practice, 31(2), 132-141.

MURRELL, P. C. (2001). The community teacher: A new framework for effective urban teaching. New York, NY: Teachers College Press.

REYES, N. (2012). Engaging English learners in community based activities to develop their literacy processes in English. Tesis de maestría, Universidad Distrital Francisco José de Caldas, Bogotá.

RUIZ, R. (1984). Language orientations. NABE Journal, 8(2), 15-34.

SCHECTER, S, SOLOMON, P.; KITTMER, L. (2003). Integrating teacher education in a community-situated school agenda. En Multilingual education in practice: Using diversity as resource. Portsmouth, NH: Heinemann.

SHARKEY, J. (2012). Community-based pedagogies and literacies in language teacher education: Promising beginnings, intriguing challenges. Íkala, 17(1). Recuperado de http://aprendeenlinea.udea.edu.co/revistas/index.php/ikala/article /view/11519

SHARKEY, J.; CLAVIJO, A. (2012). Promoting the value of local knowledge in ESL ingles teacher education through community-based field assignments. En B. Medrado y 
C. Reichmann (Eds.), Projetos e práticas na formação de professores de lingua inglesa. Paraiba: Editora Universitaria UFPB.

SHARKEY, J.; CLAVIJO, A.; RAMÍREZ, M. (2016). Developing a deeper understanding of community-based pedagogies with teachers: Learning with and from teachers in Colombia. Journal of Teacher Education, 67(3), 1-14.

SHORT, K. G., Harste, H. y Burke, C. (1996). Creating classrooms for authors and inquirers (2.a ed.). Portsmouth, NH: Heinemann.

ZEICHNER, K.; PAYNE, K. A.; BRAYKO, K. (2014). Democratizing teacher education. Journal of Teacher Education, 66(2), 122-135.

Recebido: 23/09/2019

Aceito: 2/10/2019

Publicado: 28/02/2020 